\title{
Quasiaverages, symmetry breaking and irreducible Green functions method
}

\begin{abstract}
A.L. Kuzemsky*
Bogolyubov Laboratory of Theoretical Physics, Joint Institute for Nuclear Research, 141980 Dubna, Russia

Received March 12, 2010

The development and applications of the method of quasiaverages to quantum statistical physics and to quantum solid state theory and, in particular, to quantum theory of magnetism, were considered. It was shown that the role of symmetry (and the breaking of symmetries) in combination with the degeneracy of the system was reanalyzed and essentially clarified within the framework of the method of quasiaverages. The problem of finding the ferromagnetic, antiferromagnetic and superconducting "symmetry broken" solutions of the correlated lattice fermion models was discussed within the irreducible Green functions method. A unified scheme for the construction of generalized mean fields (elastic scattering corrections) and self-energy (inelastic scattering) in terms of the equations of motion and Dyson equation was generalized in order to include the "source fields". This approach complements previous studies of microscopic theory of antiferromagnetism and clarifies the concepts of Neel sublattices for localized and itinerant antiferromagnetism and "spin-aligning fields" of correlated lattice fermions.
\end{abstract}

Key words: quantum statistical mechanics, Bogolyubov's quasiaverages, broken symmetry, quantum protectorate, quantum theory of magnetism, microscopic theory of superconductivity

PACS: 05.30.-d, 05.30.Fk, 74.20.-z, 75.10.-b

\section{Introduction}

It is well known that symmetry principles play a crucial role in physics [1, 2]. The theory of symmetry is a basic tool for understanding and formulating the fundamental notions of physics [3]. According to F. Wilczek [4], "the primary goal of fundamental physics is to discover profound concepts that illuminate our understanding of nature". It is known that symmetry is a driving force in the shaping of physical theory; moreover, the primary lesson of physics of the last century is that the secret of nature is symmetry. Every symmetry leads to a conservation law; the well known examples are the conservation of energy, momentum and electrical charge. A variety of other conservation laws can be deduced from symmetry or invariance properties of the corresponding Lagrangian or Hamiltonian of the system. According to Noether theorem, every continuous symmetry transformation under which the Lagrangian of a given system remains invariant implies the existence of a conserved function [2]. Many fundamental laws of physics apart from their detailed features possess various symmetry properties. These symmetry properties lead to certain constraints and regularities on the possible properties of matter. Thus the principles of symmetries belong to the underlying principles of physics.

It is known that when the Hamiltonian of a system is invariant under a symmetry operation, but the ground state is not, the symmetry of the system can be spontaneously broken [5]. Symmetry breaking is termed spontaneous when there is no explicit term in a Lagrangian which manifestly breaks the symmetry. Symmetries and breaking of symmetries play an important role in statistical physics, quantum field theory, physics of elementary particles, etc. [6, 7]

In physics, spontaneous symmetry breaking occurs when a system that is symmetric with respect to some symmetry group goes into a vacuum state that is not symmetric. When that happens, the system no longer appears to behave in a symmetric manner. It is a phenomenon that naturally occurs in many situations. The mechanism of spontaneous symmetry breaking is usually

*E-mail: kuzemsky@theor.jinr.ru; http://theor.jinr.ru/ kuzemsky 
understood as the mechanism responsible for the occurrence of asymmetric states in quantum systems in the thermodynamic limit and is used in various field of quantum physics [8]. The intriguing mechanism of spontaneous symmetry breaking is a unifying concept that lies at the basis of the majority of the recent developments in theoretical physics, from statistical mechanics to many-body theory and to elementary particles theory [6, 7].

It should be stressed that symmetry implies degeneracy. The greater the symmetry, the greater the degeneracy. The study of degeneracy of energy levels plays a very important role in quantum physics. It is of importance to emphasize that when spontaneous symmetry breaking takes place, the ground state of the system is degenerate. Substantial progress in the understanding of the broken symmetry concept was connected with Bogolyubov's fundamental ideas on quasiaverages [9 18]. Studies of degenerate systems led Bogolyubov in 1960-1961 to the formulation of the method of quasiaverages. This method has proved to be a universal tool for systems whose ground states become unstable under small perturbations. Thus, the role of symmetry (and the breaking of symmetries) in combination with the degeneracy of the system was reanalyzed and essentially clarified by N.N. Bogolyubov in 1960-1961. He invented and formulated a powerful innovative idea of quasiaverages in statistical mechanics [9, 10, 13, 15, 16, 18]. The very elegant work of N.N. Bogolyubov [10] has been of great importance for a deeper understanding of phase transitions, superfluidity and superconductivity, quantum theory of magnetism [19] and other fields of equilibrium and nonequilibrium statistical mechanics [10 13, 15, 16, 18, 20 23]. The concept of quasiaverages is indirectly related to the theory of phase transition. The instability of thermodynamic averages with respect to perturbations of the Hamiltonian by breaking the invariance with respect to a certain group of transformations means that there occurs a transition to an extremal state in the system. The mathematical apparatus of the method of quasiaverages includes the Bogolyubov theorem [10, 13, 18, 24] on singularities of type $1 / q^{2}$ and the Bogolyubov inequality for Green and correlation functions as a direct consequence of the method. It includes algorithms for establishing non-trivial estimates for equilibrium quasiaverages, enabling one to study the problem of ordering in statistical systems and to elucidate the structure of the energy spectrum of the underlying excited states. Thus, the Bogolyubov's idea of quasiaverages is an essential conceptual advance in modern physics.

The behaviour of many-particle systems with strong interaction is frequently quite complicated, and nonperturbative approaches are required to treat their properties. Such situations often arise in condensed matter systems. Electrical, magnetic and mechanical properties of materials are emergent collective behaviours of the underlying quantum mechanics of their electrons and constituent atoms. A principal aim of solid state physics and materials science is to elucidate this emergence. A full achievement of this goal would imply the ability to engineer a material that is optimum for any particular application. The current understanding of electrons in solids uses simplified but workable picture known as the Fermi liquid theory. This theory explains why electrons in solids can often be described in a simplified manner which appears to ignore the strong repulsive forces that electrons are known to exert on one another. There is a growing appreciation that this theory probably fails for entire classes of possibly useful materials and there is a suspicion that the failure has to do with unresolved competition between different possible emergent behaviours.

It is appropriate to note here that the emergent properties of matter were analyzed and discussed by R. Laughlin and D. Pines [25, 26] from a general point of view (see also [27]). They introduced a unifying idea of quantum protectorate. This concept also belongs to the underlying principles of physics. The idea of quantum protectorate reveals an essential difference in the behaviour of the complex many-body systems at the low-energy and high-energy scales. The existence of two scales, low-energy and high-energy, in the description of physical phenomena is used in physics, explicitly or implicitly.

On the other hand, it was recognized for many years that a strong correlation in solids exists between the motions of various particles (electrons and ions, i.e. the fermion and boson degrees of freedom) which arise from the Coulomb forces. The most interesting objects are metals and their compounds. They are invariant under the translation group of a crystal lattice and have lattice vibrations as well as electron degrees of freedom. There are many evidences for the importance 
of many-body effects in these systems. Within the semi-phenomenological theory it was suggested that the low-lying excited states of an interacting Fermi gas can be described in terms of a set of "independent quasiparticles". However, this was a phenomenological approach that did not reveal the nature of relevant interactions. An alternative way of viewing quasiparticles, more general and consistent, is through the Green function scheme of many-body theory [19, 22, 28, 29]. It becomes clear that only a thorough experimental and theoretical investigation of quasiparticle many-body dynamics of the many-particle systems can provide an answer to the relevant microscopic picture [22]. In our works, we discussed the microscopic view of a dynamic behaviour of various interacting manybody systems on a lattice [22, 30 40]. A comprehensive description of transition and rare-earth metals and alloys and other materials (as well as efficient predictions of properties of new materials) is possible only in those cases, where there is an adequate quantum-statistical theory based on the information about the electron and crystalline structures. The main theoretical problem in this direction of research, which is the essence of the quantum theory of magnetism, is to investigate and improve quantum-statistical models describing the behaviour of the complex compounds and materials in order to take into account the main features of their electronic structure, namely, their dual "band-atomic" nature [22]. The construction of a consistent theory explaining the electronic structure of these substances encounters serious difficulties when trying to describe the collectivizationlocalization duality in the behaviour of electrons. This problem appears to be extremely important, since its solution gives us a key to understanding magnetic, electronic, and other properties of this diverse group of substances. The author of the present paper investigated the suitability of the basic models with strong electron correlations and with a complex spectrum for an adequate and correct description of the dual character of electron states [22]. A universal mathematical formalism was developed for this investigation [22, 38]. It takes into account the main features of the electronic structure and allows one to describe the true quasiparticle spectrum, as well as the appearance of the magnetically ordered, superconducting, and dielectric (or semiconducting) states. With a few exceptions, diverse physical phenomena observed in compounds and alloys of transition and rare-earth metals, cannot be explained in the framework of the mean-field approximation, which overestimates the role of inter-electron correlations in computations of their static and dynamic characteristics. The realm of questions lacking a precise and definitive answer, so far, includes such extremely important (not only from a theoretical, but also from a practical point of view) problems as an adequate description of quasiparticle dynamics for quantum-statistical models in a wide range of their parameter values. The source of difficulties here lies not only in the complexity of calculations of certain dynamic properties (such as, the density of states, electrical conductivity, susceptibility, electron-phonon spectral function, the inelastic scattering cross section for slow neutrons), but also in the absence of a well-developed method for a consistent quantum-statistical analysis of a many-particle interaction in such systems. A self-consistent field approach was used in the papers [22, 30 40] for description of various dynamic characteristics of strongly correlated electronic systems. It allows one to consistently and quite compactly compute quasiparticle spectra for many-particle systems with strong interaction taking into account damping effects. The correlation effects and quasiparticle damping are the determining factors in analysing the normal properties of high-temperature superconductors, heavy fermion compounds, etc. We also formulated a general scheme for a theoretical description of electronic properties of many-particle systems taking into account strong inter-electron correlations [22, 38]. The scheme is a synthesis of the method of two-time temperature Green's functions [19, 29] and the diagram technique. An important feature of this approach is a clear-cut separation of the elastic and inelastic scattering processes in manyparticle systems (which is a highly nontrivial task for strongly correlated systems). As a result, one can construct a correct basic approximation in terms of generalized mean fields (the elastic scattering corrections), which allows one to describe the magnetically ordered or superconducting states of the system. The residual correlation effects, which are the source of quasiparticle damping, are described in terms of the Dyson equation with a formally exact representation for the mass operator.

In the present paper we will discuss some applications of the symmetry principles to quantum and statistical physics and quantum solid state theory in the light of our results on quasiparticle many-body dynamics. 


\section{Bogolyubov's quasiaverages in statistical mechanics}

In the work by N.N. Bogolyubov "Quasiaverages in problems of statistical mechanics" the innovative notion of quasiaverage [10] was introduced and applied to various problems of statistical physics. In particular, quasiaverages of Green's functions constructed from ordinary averages, degeneration of statistical equilibrium states, principle of weakened correlations, and particle pair states were considered. In this framework the $1 / q^{2}$-type properties in the theory of superfluidity of Bose and Fermi systems, the properties of basic Green functions for a Bose system in the presence of condensate, and a model with separated condensate were analyzed.

The method of quasiaverages is a constructive workable scheme for the study of the systems with spontaneous symmetry breakdown. A quasiaverage is a thermodynamic (in statistical mechanics) or vacuum (in quantum field theory) average of dynamical quantities in a specially modified averaging procedure, enabling one to take into account the effects of the state degeneracy of the system. The method provides the so-called macro-objectivation of the degeneracy in the domain of quantum statistical mechanics and in quantum physics. In statistical mechanics, under spontaneous symmetry breakdown one can, describe using the method of quasiaverages, macroscopic observables within the framework of microscopic approach.

In considering the problems of finding the eigenfunctions in quantum mechanics it is well known that the theory of perturbations should be substantially modified for the degenerate systems. In solving the problems of statistical mechanics we have always got a degenerate case due to the existence of the additive conservation laws. The traditional approach to quantum statistical mechanics [18] is based on the unique canonical quantization of classical Hamiltonians for systems with finitely many degrees of freedom together with the ensemble averaging in terms of traces involving a statistical operator $\rho$. For an operator $\mathcal{A}$ corresponding to some physical quantity $A$ the average value of $A$ will be given as

$$
\langle A\rangle_{H}=\operatorname{Tr} \rho A ; \quad \rho=\exp ^{-\beta H} / \operatorname{Tr}^{\exp ^{-\beta H}},
$$

where $H$ is the Hamiltonian of the system, $\beta=1 / k T$ is the reciprocal of the temperature.

The core of the problem lies in establishing the existence of a thermodynamic limit (such as $N / V=$ const, $V \rightarrow \infty, N=$ number of degrees of freedom, $V=$ volume) and its evaluation for the quantities of interest. Thus, in statistical mechanics the average $\langle A\rangle$ of any dynamical quantity $A$ is defined in a single-valued way. Specific problems appear in the situations with degeneracy. In quantum mechanics, if two linearly independent state vectors (wavefunctions in the Schroedinger picture) have the same energy, there is a degeneracy. In this case more than one independent state of the system corresponds to a single energy level. If the statistical equilibrium state of the system possesses lower symmetry than the Hamiltonian of the system (i.e. the situation with the symmetry breakdown), then it is necessary to supplement the averaging procedure (1) by a rule forbidding irrelevant averaging over the values of the considered macroscopic quantities for which a change is not accompanied by a change in energy. This is achieved by introducing quasiaverages, that is, averages over the Hamiltonian $H_{\nu \vec{e}}$ supplemented by infinitesimally-small terms that violate the additive conservations laws $H_{\nu \vec{e}}=H+\nu(\vec{e} \cdot \vec{M}),(\nu \rightarrow 0)$. Thermodynamic averaging may turn out to be unstable with respect to such a change of the original Hamiltonian, which is another indication of degeneracy of the equilibrium state. According to Bogolyubov [10], the quasiaverage of a dynamical quantity $A$ for the system with the Hamiltonian $H_{\nu \vec{e}}$ is defined as the limit

$$
\prec A \succ=\lim _{\nu \rightarrow 0}\langle A\rangle_{\nu \vec{e}},
$$

where $\langle A\rangle_{\nu \vec{e}}$ denotes the ordinary average taken over the Hamiltonian $H_{\nu \vec{e}}$, containing the small symmetry-breaking terms introduced by the inclusion parameter $\nu$, that vanish as $\nu \rightarrow 0$ after the passage to thermodynamic limit $V \rightarrow \infty$. It is important to note that in this equation the limits cannot be interchanged. Thus, the existence of degeneracy is reflected directly in the quasiaverages by their dependence upon the arbitrary unit vector $\vec{e}$. It is also clear that

$$
\langle A\rangle=\int \prec A \succ \mathrm{d} \vec{e} .
$$


According to definition (3), the ordinary thermodynamic average is obtained by extra averaging of the quasiaverage over the symmetry-breaking group. Thus, quasiaverages are more convenient and more physical than ordinary averages [18] in order to describe the case of a degenerate state of statistical equilibrium. The latter are the same quasiaverages only averaged over all the directions $\vec{e}$.

It is necessary to stress, that the starting point for Bogolyubov's work [10] was an investigation of additive conservation laws and selection rules, continuing and developing the approach by P. Curie for derivation of selection rules for physical effects (see also [6]). Bogolyubov demonstrated that in the cases when the state of statistical equilibrium is degenerate, as in the case of the Heisenberg ferromagnet, one can remove the degeneracy of equilibrium states with respect to the group of spin rotations by including an additional noninvariant term $\nu M_{z} V$ with an infinitely small $\nu$ into the Hamiltonian $H$. For the Heisenberg ferromagnet, the ordinary averages should be invariant with regard to the spin rotation group. The corresponding quasiaverages possess only the property of covariance. Thus, the quasiaverages do not follow the same selection rules as those which govern ordinary averages, due to their invariance with regard to the spin rotation group. It is clear that the unit vector $\vec{e}$, i.e., the direction of magnetization $\vec{M}$ vector, characterizes the degeneracy of the considered state of statistical equilibrium. In order to remove the degeneracy one should fix the direction of the unit vector $\vec{e}$. It can be chosen to be along the $z$ direction. Then, all the quasiaverages will be definite numbers. This is the kind that one usually deals with in the theory of ferromagnetism.

The value of a quasi-average (2) may depend on the concrete structure of the additional term $\Delta H=H_{\nu}-H$, if the dynamical quantity to be averaged is not invariant with respect to the symmetry group of the original Hamiltonian $H$. For a degenerate state, the limit of ordinary averages (3), as the inclusion parameters $\nu$ of the sources tend to zero in an arbitrary fashion, may not exist. For a complete definition of quasiaverages it is necessary to indicate the manner in which these parameters tend to zero in order to ensure convergence [15]. On the other hand, in order to remove degeneracy it suffices, in the construction of $H$, to violate only those additive conservation laws whose switching leads to instability of the ordinary average. Thus, in terms of quasiaverages, the selection rules for the correlation functions [11, 18] that are not relevant are those restricted by these conservation laws.

By using $H_{\nu}$, we define the state $\omega(A)=\langle A\rangle_{\nu}$ and then let $\nu$ tend to zero (after passing to the thermodynamic limit) [10, 11, 18]. If all averages $\omega(A)$ get infinitely small increments under infinitely small perturbations $\nu$, this means that the state of statistical equilibrium under consideration is nondegenerate [10,11, 18]. However, if some states have finite increments as $\nu \rightarrow 0$, then the state is degenerate. In this case, instead of ordinary averages $\langle A\rangle_{H}$, one should introduce the quasiaverages (2), for which the usual selection rules do not hold.

The method of quasiaverages is directly related to the principle of weakening of the correlation [10, 11, 18] in many-particle systems. According to this principle, the notion of the weakening of the correlation, known in statistical mechanics [10, 11, 18], in the case of state degeneracy should be interpreted in the sense of quasiaverages [11].

The quasiaverages may be obtained from the ordinary averages by using the cluster property which was formulated by Bogolyubov [11]. This was first done when deriving the Boltzmann equations from the chain of equations for distribution functions, and in the investigation of the model Hamiltonian in the theory of superconductivity [9, 10, 13, 16, 18. To demonstrate this let us consider the averages (quasiaverages) of the form

$$
F\left(t_{1}, x_{1}, \ldots t_{n}, x_{n}\right)=\left\langle\ldots \Psi^{\dagger}\left(t_{1}, x_{1}\right) \ldots \Psi\left(t_{j}, x_{j}\right) \ldots\right\rangle,
$$

where the number of creation operators $\Psi^{\dagger}$ may be not equal to the number of annihilation operators $\Psi$. We fix times and split the arguments $\left(t_{1}, x_{1}, \ldots t_{n}, x_{n}\right)$ into several clusters $\left(\ldots, t_{\alpha}, x_{\alpha}, \ldots\right)$, $\ldots,\left(\ldots, t_{\beta}, x_{\beta}, \ldots\right)$. Then, it is reasonable to assume that the distances between all clusters $\left|x_{\alpha}-x_{\beta}\right|$ tend to infinity. Then, according to the cluster property, the average value (4) tends to the product of averages of collections of operators with the $\operatorname{arguments}\left(\ldots, t_{\alpha}, x_{\alpha}, \ldots\right), \ldots$, $\left(\ldots, t_{\beta}, x_{\beta}, \ldots\right)$

$$
\lim _{\left|x_{\alpha}-x_{\beta}\right| \rightarrow \infty} F\left(t_{1}, x_{1}, \ldots t_{n}, x_{n}\right)=F\left(\ldots, t_{\alpha}, x_{\alpha}, \ldots\right) \ldots F\left(\ldots, t_{\beta}, x_{\beta}, \ldots\right) .
$$


For equilibrium states with small densities and short-range potential, the validity of this property can be proved [18]. For a general case, the validity of the cluster property has not yet been proved. Bogolyubov formulated it not only for ordinary averages but also for quasiaverages, i.e., for anomalous averages, too. It works for many important models, including the models of superfluidity [13, 18] and superconductivity [13, 18, 41, 42] ( see also [43-45]).

To illustrate this statement consider Bogolyubov's theory of a Bose-system with separated condensate, which is given by the Hamiltonian [13, 18]

$$
\begin{aligned}
H_{\Lambda}= & \int_{\Lambda} \Psi^{\dagger}(x)\left(-\frac{\Delta}{2 m}\right) \Psi(x) \mathrm{d} x-\mu \int_{\Lambda} \Psi^{\dagger}(x) \Psi(x) \mathrm{d} x \\
& +\frac{1}{2} \int_{\Lambda^{2}} \Psi^{\dagger}\left(x_{1}\right) \Psi^{\dagger}\left(x_{2}\right) \Phi\left(x_{1}-x_{2}\right) \Psi\left(x_{2}\right) \Psi\left(x_{1}\right) \mathrm{d} x_{1} \mathrm{~d} x_{2} .
\end{aligned}
$$

This Hamiltonian can be also written in the following form

$$
\begin{aligned}
H_{\Lambda}= & H_{0}+H_{1}=\int_{\Lambda} \Psi^{\dagger}(q)\left(-\frac{\Delta}{2 m}\right) \Psi(q) \mathrm{d} q \\
& +\frac{1}{2} \int_{\Lambda^{2}} \Psi^{\dagger}(q) \Psi^{\dagger}\left(q^{\prime}\right) \Phi\left(q-q^{\prime}\right) \Psi\left(q^{\prime}\right) \Psi(q) \mathrm{d} q \mathrm{~d} q^{\prime} .
\end{aligned}
$$

Here, $\Psi(q)$, and $\Psi^{\dagger}(q)$ are the operators of annihilation and creation of bosons. They satisfy the canonical commutation relations

$$
\left[\Psi(q), \Psi^{\dagger}\left(q^{\prime}\right)\right]=\delta\left(q-q^{\prime}\right) ; \quad\left[\Psi(q), \Psi\left(q^{\prime}\right)\right]=\left[\Psi^{\dagger}(q), \Psi^{\dagger}\left(q^{\prime}\right)\right]=0 .
$$

The system of bosons is contained in the cube $A$ with the edge $L$ and volume $V$. It was assumed that it satisfies periodic boundary conditions and the potential $\Phi(q)$ is spherically symmetric and proportional to the small parameter. It was also assumed that, at temperature zero, a certain macroscopic number of particles having a nonzero density is located in the state with momentum zero. The operators $\Psi(q)$, and $\Psi^{\dagger}(q)$ can be represented in the form

$$
\Psi(q)=a_{0} / \sqrt{V} ; \quad \Psi^{\dagger}(q)=a_{0}^{\dagger} / \sqrt{V}
$$

where $a_{0}$ and $a_{0}^{\dagger}$ are the operators of annihilation and creation of particles with momentum zero.

To explain the phenomenon of superfluidity, one should calculate the spectrum of the Hamiltonian, which is quite a difficult problem. Bogolyubov suggested the idea of approximate calculation of the spectrum of the ground state and its elementary excitations based on the physical nature of superfluidity. His idea consists of a few assumptions. The main assumption is that at temperature zero, the macroscopic number of particles (with nonzero density) has the momentum zero. Therefore, in the thermodynamic limit, the operators $a_{0} / \sqrt{V}$ and $a_{0}^{\dagger} / \sqrt{V}$ commute

$$
\lim _{V \rightarrow \infty}\left[a_{0} / \sqrt{V}, a_{0}^{\dagger} / \sqrt{V}\right]=\frac{1}{V} \rightarrow 0
$$

and are $c$-numbers. Hence, the operator of the number of particles $N_{0}=a_{0}^{\dagger} a_{0}$ is a $c$-number, too. It is worth noting that the Hamiltonian (7) is invariant under the gauge transformation $\tilde{a}_{k}=\exp (\mathrm{i} \varphi) a_{k}, \tilde{a}_{k}^{\dagger}=\exp (-\mathrm{i} \varphi) a_{k}^{\dagger}$, where $\varphi$ is an arbitrary real number. Therefore, the averages $\left\langle a_{0} / \sqrt{V}\right\rangle$ and $\left\langle a_{0}^{\dagger} / \sqrt{V}\right\rangle$ should vanish. However, this contradicts the assumption that $a_{0} / \sqrt{V}$ and $a_{0}^{\dagger} / \sqrt{V}$ must become $c$-numbers in the thermodynamic limit. Moreover, it should be taken into account that $a_{0}^{\dagger} a_{0} / V=N_{0} / V \neq 0$ which implies that $a_{0} / \sqrt{V}=N_{0} \exp (\mathrm{i} \alpha) / \sqrt{V} \neq 0$ and $a_{0}^{\dagger} / \sqrt{V}=$ $N_{0} \exp (-\mathrm{i} \alpha) / \sqrt{V} \neq 0$, where $\alpha$ is an arbitrary real number. This contradiction may be overcome if we assume that the eigenstates of the Hamiltonian are degenerate and not invariant under gauge transformations, i. e., that a spontaneous breaking of symmetry takes place. 
Thus, the averages $\left\langle a_{0} / \sqrt{V}\right\rangle$ and $\left\langle a_{0}^{\dagger} / \sqrt{V}\right\rangle$, which are nonzero under spontaneously broken gauge invariance, are called anomalous averages or quasiaverages. This innovative idea by Bogolyubov penetrates deeply into the modern quantum physics. The systems with spontaneously broken symmetry are studied using the transformation of the operators of the form

$$
\Psi(q)=a_{0} / \sqrt{V}+\theta(q) ; \quad \Psi^{\dagger}(q)=a_{0}^{\dagger} / \sqrt{V}+\theta^{*}(q),
$$

where $a_{0} / \sqrt{V}$ and $a_{0}^{\dagger} / \sqrt{V}$ are the numbers first introduced by Bogolyubov in 1947 in his investigation of the phenomenon of superfluidity [10, 13, 18]. The main conclusion was made that for the systems with spontaneously broken symmetry, the quasiaverages should be studied instead of the ordinary averages. It turns out that the long-range order appears not only in the system of Bose-particles but also in all systems with spontaneously broken symmetry. The above outlined Bogolyubov's papers anticipated the methods of investigation of the systems with spontaneously broken symmetry for many years.

As mentioned above, in order to explain the phenomenon of superfluidity, Bogolyubov assumed that the operators $a_{0} / \sqrt{V}$ and $a_{0}^{\dagger} / \sqrt{V}$ become $c$-numbers in the thermodynamic limit. This statement was rigorously proved in the papers by Bogolyubov and by some other authors. Bogolyubov's proof was based on the study of the equations for two-time Green's functions [29] and on the assumption that the cluster property holds. It was proved that the solutions of equations for Green's functions for the system with Hamiltonian (7) coincide with the solutions of the equations for the system with the same Hamiltonian in which the operators $a_{0} / \sqrt{V}$ and $a_{0}^{\dagger} / \sqrt{V}$ are replaced by numbers. These numbers should be determined from the condition of minimum for free energy. Since all the averages in both systems coincide, their free energies coincide, too.

It is worth noting that the validity of the replacement of the operators $a_{0}$ and $a_{0}^{\dagger}$ by $c$-numbers in the thermodynamic limit was confirmed in numerous subsequent publications of various authors. Thus, Bogolyubov's 1947 analysis of the many-body Hamiltonian by means of a $c$-number substitution for the most relevant operators in the problem, the zero-momentum mode operators, was rigorously justified. Since the Bogolyubov's 1947 analysis is one of the key developments in the theory of the Bose gas, especially the theory of low density gases currently at the forefront of experiment 43 45], this result is of importance for the legitimation of that theory. Additional arguments were given in the study, where the Bose-Einstein condensation and spontaneous $U(1)$ symmetry breaking were investigated on the basis of Bogolyubov's truncated Hamiltonian $H_{\mathrm{B}}$ for a weakly interacting Bose system, and adding a $U(1)$ symmetry breaking term $\sqrt{V}\left(\lambda a_{0}+\lambda^{*} a_{0}^{\dagger}\right)$ to $H_{\mathrm{B}}$, It was also shown, by using the coherent state theory and the mean-field approximation rather than the $c$-number approximations, that the Bose-Einstein condensation occurs if and only if the $U(1)$ symmetry of the system is spontaneously broken. The real ground state energy and justification of the Bogolyubov $c$-number substitution were provided by solving the Schroedinger eigenvalue equation and using the self-consistent condition. Thus, the Bogolyubov $c$-number substitutions were fully correct and it is obvious that the symmetry breaking causes the displacement of the condensate state.

The concept of quasiaverages was introduced by Bogolyubov on the basis of an analysis of manyparticle systems with a degenerate statistical equilibrium state. Such states are inherent to various physical many-particle systems [13, 18]. Those are liquid helium in the superfluid phase, metals in the superconducting state, magnets in the ferromagnetically ordered state, liquid crystal states, the states of superfluid nuclear matter, etc. (for a review, see [22, 46]). In case of superconductivity, the source $\nu \sum_{k} v(k)\left(a_{k \uparrow}^{\dagger} a_{-k \downarrow}^{\dagger}+a_{-k \downarrow} a_{k \uparrow}\right)$ was inserted in the BCS-Bogolyubov Hamiltonian, and the quasiaverages were defined using the Hamiltonian $H_{\nu}$. In the general case, the sources are introduced to remove degeneracy. If infinitesimal sources give infinitely small contributions to the averages, this means that there is no corresponding degeneracy, and there is no reason to insert sources into the Hamiltonian. Otherwise, the degeneracy takes place, and it is removed by the sources. The ordinary averages can be obtained from quasiaverages by averaging with respect to the parameters that characterize the degeneracy.

N. N. Bogolyubov (Jr.) [15] considered some features of quasiaverages for model systems with four-fermion interaction. He discussed the treatment of certain three-dimensional model systems 
which can be solved exactly. To this end, a new effective way of defining quasiaverages for the systems under consideration was proposed.

Peletminskii and Sokolovskii [47] have found general expressions for the operators of the flux densities of physical variables in terms of the density operators of these variables. The method of quasiaverages and the expressions found for the flux operators were used to obtain the averages of these operators in terms of thermodynamic potential in a state of statistical equilibrium of a superfluid liquid.

Vozyakov [4] reformulated the theory of quantum crystals in terms of quasiaverages. He analyzed a Bose system with periodic distribution of particles which simulates an ensemble in which the particles cannot be regarded as vibrating independently about a position of equilibrium lattice sites. With allowance for macroscopic filling of the states corresponding to the distinguished symmetry, a calculation was made of an excitation spectrum in which there exists a collective branch of gapless type.

Peregoudov [49] discussed the effective potential method, used in quantum field theory to study spontaneous symmetry breakdown, from the point of view of Bogolyubov's quasiaveraging procedure. It was shown that the effective potential method is a disguised type of this procedure. The catastrophe theory approach to the study of phase transitions was discussed and the existence of the potentials used in that approach was proved from the statistical point of view. It was shown that in the ease of broken symmetry, the nonconvex effective potential is not a Legendre transform of the generating functional for connected Green's functions. Instead, it is a part of the potential used in catastrophe theory. The relationship between the effective potential and the Legendre transform of the generating functional for connected Green's functions is given by Maxwell's rule. A rigorous rule for evaluating quasiaveraged quantities within the framework of the effective potential method was established.

N.N. Bogolyubov (Jr.) with M. Yu. Kovalevsky and co-authors 50] developed a statistical approach for solving the problem of classification of equilibrium states in condensed media with spontaneously broken symmetry based on the quasiaverage concept. Classification of equilibrium states of condensed media with spontaneously broken symmetry was carried out. The generators of residual and spatial symmetries were introduced and equations of classification for the order parameter were found. Conditions of residual symmetry and spatial symmetry were formulated. The connection between these symmetry conditions and equilibrium states of various media with tensor order parameter was found out. An analytical solution of the problem of classification of equilibrium states for superfluid media, liquid crystals and magnets with tensor order parameters was obtained. Superfluid ${ }^{3} \mathrm{He}$, liquid crystals, quadrupolar magnetics were considered in detail. Possible homogeneous and heterogeneous states were found out. Discrete and continuous thermodynamic parameters, which define an equilibrium state, allowable form of order parameter, residual symmetry, and spatial symmetry generators were established. This approach, which is alternative to the well-known Ginzburg-Landau method, does not contain any model assumptions concerning the form of the free energy as functional of the order parameter and does not employ the requirement of temperature closeness to the point of phase transition. For all the investigated cases they found the structure of the order parameters and the explicit forms of generators of residual and spatial symmetries. Under certain restrictions they established the form of the order parameters in case of spins $0,1 / 2,1$ and proposed a physical interpretation of the studied degenerate states of condensed media.

\section{Quantum protectorate}

The "quantum protectorate" concept was formulated in paper 25]. Its inventors, R. Laughlin and D. Pines, discussed the most fundamental principles of matter description in the widest sense of this word. They formulated their main thesis: emergent physical phenomena, which are regulated by higher physical principles, have a certain property, typical of these phenomena only. This property is their insensitivity to microscopic description. For instance, the crystalline state is the simplest known example of a quantum protectorate, a stable state of matter whose generic 
low-energy properties are determined by a higher organizing principle and nothing else. There are many other examples [25]. These quantum protectorates, with their associated emergent behaviour, provide us with explicit demonstrations that the underlying microscopic theory can easily have no measurable consequences whatsoever at low energies. The nature of the underlying theory is unknowable until one raises the energy scale sufficiently to escape protection. The existence of two scales, the low-energy and high-energy scales, relevant to the description of magnetic phenomena was stressed by the author of the present work in the papers [22, 51, 52, which were devoted to comparative analysis of localized and band models of quantum theory of magnetism. It was shown there that the low-energy spectrum of magnetic excitations in the magnetically-ordered solid bodies corresponds to a hydrodynamic pole $(\vec{k}, \omega \rightarrow 0)$ in the generalized spin susceptibility $\chi$, which is present in the Heisenberg, Hubbard, and the combined $s-d$ model. In the Stoner band model the hydrodynamic pole is absent, there are no spin waves there. At the same time, the Stoner single-particle's excitations are absent in the Heisenberg model's spectrum. The Hubbard model with narrow energy bands contains both types of excitations: the collective spin waves (the low-energy spectrum) and Stoner single-particle's excitations (the high-energy spectrum). This is a big advantage and flexibility of the Hubbard model in comparison to the Heisenberg model. The latter, nevertheless, is a very good approximation to the realistic behaviour in the limit $\vec{k}, \omega \rightarrow 0$, the domain where the hydrodynamic description is applicable, that is, for long wavelengths and low energies. The quantum protectorate concept was applied to the quantum theory of magnetism by the present author in paper [52], where a criterion of applicability of models of the quantum theory of magnetism to the description of concrete substances was formulated. The criterion is based on the analysis of the model's low-energy and high-energy spectra. Therein a detailed analysis was carried out of the idea of quantum protectorate [25] in the context of quantum theory of magnetism [52]. It was suggested that the difficulties in the formulation of quantum theory of magnetism at the microscopic level, that are related to the choice of relevant models, can be better understood in the light of the quantum protectorate concept [52]. We argued that the difficulties in the formulation of adequate microscopic models of electron and magnetic properties of materials are intimately related to dual, itinerant and localized behaviour of electrons [51]. We formulated a criterion for determining the basic picture that best describes this dual behaviour. The main suggestion was that quasiparticle excitation spectra might provide distinctive signatures and good criteria for the appropriate choice of the relevant model. A broad class of the problems of condensed matter physics [22, 23] in the fields of the the magnetism and superconductivity of complex materials were reconsidered in relation to these ideas.

\section{Irreducible Green functions method}

It was shown above that it becomes clear that only a thorough experimental and theoretical investigation of quasiparticle many-body dynamics of the many-particle systems can provide an answer to the relevant microscopic picture. Many-particle systems where the interaction is strong have often got a complicated behaviour, and require nonperturbative approaches to the treatment of their properties. There are many different approaches to the construction of generalized mean-field approximations; however, all of them have a special-case character. The method of irreducible Green functions (IGF) allows one to tackle this problem in a more systematic fashion. In order to clarify this statement, let us briefly consider the main ideas of the IGF approach that allows one to describe completely quasiparticle spectra with damping in a very natural way. When working with infinite hierarchies of equations for Green functions, the main problem lies in finding the methods for their efficient decoupling, with the aim of obtaining a closed system of equations, which determine the Green functions. A decoupling approximation should be chosen individually for every particular problem, taking into account its character. This "individual approach" is the source of critique for being too non-transparent, which sometimes appears in the papers using the causal Green functions and diagram technique. However, the ambiguities are also present in the diagram technique, when the choice of an appropriate approximation is made there. The decision, which diagrams one has to sum up, is obvious only for a narrow range of relatively simple problems. In the paper 53. 
devoted to Bose-systems, and in the papers by the author of present work [30, 31, 38] devoted to Fermi systems it was shown that for a wide range of problems in statistical mechanics and theory of condensed matter one can outline a fairly systematic recipe for constructing approximate solutions in the framework of irreducible Green's functions method. Within this approach one can look from a unified point of view at the main problems of fundamental character arising in the method of two-time temperature Green functions. The method of irreducible Green functions is a useful reformulation of the ordinary Bogolyubov-Tyablikov method of equations of motion [19, 29].

We reformulated the two-time Green functions method [19, 30, 31, 38] to the form which is especially adjusted to correlated fermion systems on a lattice and systems with complex spectra. A very important concept of the whole method is the generalized mean fields (GMFs), as it was formulated in [22, 38]. These GMFs have a complicated structure for a strongly correlated case and complex spectra, and are not reduced to the functional of mean densities of the electrons or spins when one calculates excitation spectra at finite temperatures.

To clarify the foregoing, let us consider a retarded Green function of the form [19]

$$
G^{\mathrm{r}}=\left\langle\left\langle A(t), A^{\dagger}\left(t^{\prime}\right)\right\rangle\right\rangle=-\mathrm{i} \theta\left(t-t^{\prime}\right)\left\langle\left[A(t) A^{\dagger}\left(t^{\prime}\right)\right]_{\eta}\right\rangle, \quad \eta= \pm 1 .
$$

As an introduction to the concept of IGF, let us describe the main ideas of this approach in a symbolic and simplified form. To calculate the retarded Green function $G\left(t-t^{\prime}\right)$, let us write down the equation of motion for it

$$
\omega G(\omega)=\left\langle\left[A, A^{\dagger}\right]_{\eta}\right\rangle+\left\langle\left\langle[A, H]_{-} \mid A^{\dagger}\right\rangle\right\rangle_{\omega} .
$$

Here we use the notation $\left\langle\left\langle A(t), A^{\dagger}\left(t^{\prime}\right)\right\rangle\right\rangle$ for the time-dependent Green function and $\left\langle\left\langle A \mid A^{\dagger}\right\rangle\right\rangle_{\omega}$ for its Fourier transform [19]. The notation $[A, B]_{\eta}$ refers to commutation and anticommutation, depending on the value of $\eta= \pm$. The essence of the method is as follows 38 ]. It is based on the notion of the "IRREDUCIBLE" parts of Green functions (or the irreducible parts of the operators, $A$ and $A^{\dagger}$, out of which the Green function is constructed) in terms of which it is possible, without recourse to a truncation of the hierarchy of equations for the Green functions, to write down the exact Dyson equation and to obtain an exact analytic representation for the self-energy operator. By definition, we introduce the irreducible part (ir) of the Green function

$$
{ }^{(i r)}\left\langle\left\langle[A, H]_{-} \mid A^{\dagger}\right\rangle\right\rangle=\left\langle\left\langle[A, H]_{-}-z A \mid A^{\dagger}\right\rangle\right\rangle .
$$

The unknown constant $\mathrm{z}$ is defined by the condition (or constraint)

$$
\left\langle\left[[A, H]_{-}^{(\mathrm{ir})}, A^{\dagger}\right]_{\eta}\right\rangle=0,
$$

which is an analogue of the orthogonality condition in the Mori formalism [54, 55]. Let us emphasize that due to the complete equivalence of the definition of the irreducible parts for the Green functions $\left.{ }^{(\text {ir) }}\left\langle\left\langle[A, H]_{-} \mid A^{\dagger}\right\rangle\right\rangle\right)$ and operators $\left.{ }^{(\text {ir) }}[A, H]_{-}\right) \equiv\left([A, H]_{-}\right)^{\text {(ir) }}$ we will use the both notations freely ( ${ }^{(i r)}\langle\langle A \mid B\rangle\rangle$ is the same as $\left.\left\langle\left\langle(A)^{(\mathrm{ir})} \mid B\right\rangle\right\rangle\right)$. A preferable choice of one notation over the other is determined by the brevity and clarity of notation itself. From the condition (15) one can find

$$
z=\frac{\left\langle\left[[A, H]_{-}, A^{\dagger}\right]_{\eta}\right\rangle}{\left\langle\left[A, A^{\dagger}\right]_{\eta}\right\rangle}=\frac{M_{1}}{M_{0}} .
$$

Here $M_{0}$ and $M_{1}$ are the zeroth and first order moments of the spectral density. Therefore, the irreducible Green functions are defined so that they cannot be reduced to the lower-order ones by any kind of decoupling. It is worth noting that the term "irreducible" in a group theory means a representation of a symmetry operation that cannot be expressed in terms of lower dimensional representations. Irreducible (or connected ) correlation functions are known in statistical mechanics. In the diagrammatic approach, the irreducible vertices are defined as graphs that do not contain inner parts connected by the $G^{0}$-line. With the aid of the definition (14) these concepts are expressed in terms of retarded and advanced Green functions. The procedure extracts all relevant 
(for the problem under consideration) mean-field contributions and puts them into the generalized mean-field Green function which is defined here as

$$
G^{0}(\omega)=\frac{\left\langle\left[A, A^{\dagger}\right]_{\eta}\right\rangle}{(\omega-z)}
$$

To calculate the IGF (ir) $\left\langle\left\langle[A, H]_{-}(t), A^{\dagger}\left(t^{\prime}\right)\right\rangle\right\rangle$ in (13), we have to write the equation of motion for it after differentiation with respect to the second time variable $t^{\prime}$. The condition of orthogonality (15) removes the inhomogeneous term from this equation and is a very crucial point of the whole approach. If one introduces the irreducible part for the right-hand side operator, as discussed above for the "left" operator, the equation of motion (13) can be exactly rewritten in the following form:

$$
G=G^{0}+G^{0} P G^{0} .
$$

The scattering operator $P$ is given by

$$
P=\left(M_{0}\right)^{-1}\left({ }^{(i r)}\left\langle\left\langle[A, H]_{-} \mid\left[A^{\dagger}, H\right]_{-}\right\rangle\right\rangle^{(\text {ir })}\right)\left(M_{0}\right)^{-1}
$$

The structure of equation (18) enables us to determine the self-energy operator $M$ by analogy with the diagram technique

$$
P=M+M G^{0} P
$$

Here we used the notation $M$ for self-energy (mass operator in quantum field theory). From the definition (20) it follows that the self-energy operator $M$ is defined as a proper (in the diagrammatic language, "connected") part of the scattering operator $M=(P)^{p}$. As a result, we obtain the exact Dyson equation for the thermodynamic double-time Green functions

$$
G=G^{0}+G^{0} M G
$$

The difference between $P$ and $M$ can be regarded as two different solutions of two integral equations (18) and (21). However, from the Dyson equation (21) only the full GF is seen to be expressed as a formal solution of the form

$$
G=\left[\left(G^{0}\right)^{-1}-M\right]^{-1} .
$$

Equation (22) can be regarded as an alternative form of the Dyson equation (21) and the definition of $M$ provides that the generalized mean-field GF $G^{0}$ is specified. On the contrary, for the scattering operator $P$, instead of the property $G^{0} G^{-1}+G^{0} M=1$, one has the property

$$
\left(G^{0}\right)^{-1}-G^{-1}=P G^{0} G^{-1}
$$

Thus, the very functional form of the formal solution (22) precisely determines the difference between $P$ and $M$.

Thus, by introducing irreducible parts of GF (or irreducible parts of the operators, out of which the GF is constructed) the equation of motion (13) for the GF can be exactly transformed (but using the orthogonality constraint (15)) into the Dyson equation for the double-time thermal GF (21). This result is very remarkable because the traditional form of the GF method does not include this point. Notice that all quantities thus considered are exact. Approximations can be generated not by truncating the set of coupled equations of motions but by a specific approximation of the functional form of the mass operator $M$ within a self-consistent scheme expressing $M$ in terms of the initial GF

$$
M \approx F[G] .
$$

Different approximations are relevant to different physical situations. The projection operator technique has essentially the same philosophy. But with using the constraint (15) in our approach we emphasize the fundamental and central role of the Dyson equation for calculation of singleparticle properties of many-body systems. The problem of reducing the whole hierarchy of equations involving higher-order GFs by a coupled nonlinear set of integro-differential equations connecting 
the single-particle GF to the self-energy operator is rather nontrivial. A characteristic feature of these equations is that besides the single-particle GF they also involve higher-order GF. The irreducible counterparts of the GFs, vertex functions, serve to correctly identify the self-energy as

$$
M=G_{0}^{-1}-G^{-1} .
$$

The integral form of the Dyson equation (21) provides $M$ with the physical meaning of a nonlocal and energy-dependent effective single-particle potential. This meaning can be verified for the exact self-energy using the diagrammatic expansion for the causal GF.

It is important to note that for the retarded and advanced GFs, the notion of the proper part $M=(P)^{p}$ is symbolic in nature [22, 38]. In a certain sense, it is possible to say that it is defined here by analogy with the irreducible many-particle $T$-matrix. Furthermore, by analogy with the diagrammatic technique, we can also introduce the proper part defined as a solution to the integral equation (20). These analogues allow us to better understand the formal structure of the Dyson equation for the double-time thermal GF, but only in a symbolic form. However, because of the identical form of the equations for GFs for all three types (advanced, retarded, and causal), we can convert our calculations to causal GF at each stage of calculations and, thereby, confirm the substantiated nature of definition (20). We, therefore, should speak of an analogy of the Dyson equation. Hereafter, we drop this stipulating, since it does not cause any misunderstanding. In a sense, the IGF method is a variant of the Gram-Schmidt orthogonalization procedure 38].

It should be emphasized that the scheme presented above gives just a general idea of the IGF method. A more exact explanation why one should not introduce the approximation already in $P$, instead of having to work out $M$, is given below when working out the application of the method to specific problems.

The general philosophy of the IGF method is in the separation and identification of elastic scattering effects and inelastic ones. This latter point is quite often underestimated, and both effects are mixed. However, as far as the right definition of quasiparticle damping is concerned, the separation of elastic and inelastic scattering processes is believed to be crucially important for many-body systems with complicated spectra and strong interaction.

From a technical point of view, the elastic GMF renormalizations can exhibit quite a nontrivial structure. To obtain this structure correctly, one should construct a full GF from a complete algebra of relevant operators and develop a special projection procedure for higher-order GFs, in accordance with a given algebra. Then a natural question arises how to select the relevant set of operators $\left\{A_{1}, A_{2}, \ldots A_{n}\right\}$ describing the "relevant degrees of freedom". The above consideration suggests an intuitive and heuristic way to the suitable procedure arising from an infinite chain of equations of motion (13). Let us consider the column

$$
\left(\begin{array}{c}
A_{1} \\
A_{2} \\
\vdots \\
A_{n}
\end{array}\right)
$$

where

$$
A_{1}=A, \quad A_{2}=[A, H], \quad A_{3}=[[A, H], H], \ldots A_{n}=[[\ldots[A, \underbrace{H] \ldots H}_{n}] .
$$

Then, the most general possible Green function can be expressed as a matrix

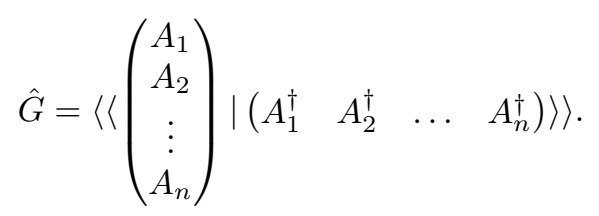

This generalized Green function describes the one-, two-, and $n$-particle dynamics. The equation of motion for it includes, as a particular case, the Dyson equation for single-particle Green function, 
and the Bethe-Salpeter equation which is the equation of motion for the two-particle Green function and which is an analogue of the Dyson equation, etc. The corresponding reduced equations should be extracted from the equation of motion for the generalized Green function with the aid of special techniques such as the projection method and similar techniques. This should be a final goal towards a real understanding of the true many-body dynamics. At this point, it is worthwhile to underline that the above discussion is a heuristic scheme only, rather than a straightforward recipe. The specific method of introducing the IGFs depends on the form of operators $A_{n}$, the type of the Hamiltonian, and conditions of the problem. Here a sketchy form of the IGF method was presented. The aim was to introduce the general scheme and to lay the groundwork for generalizations. We demonstrated in [22, 38] that the IGF method is a powerful tool for describing the quasiparticle excitation spectra, allowing for a more profound understanding of elastic and inelastic quasiparticle scattering effects and the corresponding aspects of damping and finite lifetimes. In a certain sense, it provides a clear link between the equation-of-motion approach and the diagrammatic methods due to derivation of the Dyson equation. Moreover, due to the fact that it enables an approximate treatment of self-energy effects at the final stage, it yields a systematic way of constructing approximate solutions.

\section{Effective and generalized mean fields}

The most common technique for studying the subject of interacting many-particle systems is to use the mean field theory. This approximation was especially popular in the theory of magnetism [19, 22, 38]. To calculate the susceptibility and other characteristic functions of a system of localized magnetic moments, with a given interaction Hamiltonian, the approximation, termed the "molecular field approximation" was widely used. However, it is not an easy task to give a formal unified definition of the mean field. In a sense, the mean field is the umbrella term for a variety of theoretical methods of reducing the many-particle problem to the single-particle one. Mean field theory, that approximates the behaviour of a system by ignoring the effect of fluctuations and those spin correlations which dominate the collective properties of the ferromagnet usually provides a starting and estimating point only, for studying phase transitions. The mean field theories miss important features of the dynamics of a system. The main intention of the mean field theories, starting with the works of J. D. van der Waals and P. Weiss, is to take into account the cooperative behaviour of a large number of particles. It is well known that earlier theories of phase transitions based on the ideas of van der Waals and Weiss lead to predictions which are qualitatively at variance with results of measurements near the critical point. Other variants of simplified mean field theories such as the Hartree-Fock theory for electrons in atoms, lead to discrepancies of various kinds too. It is therefore natural to analyze the reasons for such drawbacks of earlier variants of the mean field theories.

A number of effective field theories which are improved versions of the "molecular field approximation" were proposed. In our papers [22, 30, 31, 38] we stressed a specificity of strongly correlated many-particle systems on a lattice contrary to continuum (uniform) systems. The earlier concepts of molecular field were described in terms of a functional of mean magnetic moments (in magnetic terminology) or mean particle densities. The corresponding mean-field functional $F\left[\langle n\rangle,\left\langle S^{z}\right\rangle\right]$ describes the uniform mean field. Actually, the Weiss model was not based on discrete "spins" as is well known, but the uniformity of the mean internal field was the most essential feature of the model. In the modern language, one should assume that the interaction between atomic spins $S_{i}$ and its neighbors is equivalent to a mean (or molecular) field, $M_{i}=\chi_{0}\left[h_{i}^{(\mathrm{ext})}+h_{i}^{(\mathrm{mf})}\right]$ and that the molecular field $h_{i}^{(\mathrm{mf})}$ is of the form $h^{(\mathrm{mf})}=\sum_{i} J\left(R_{j i}\right)\left\langle S_{i}\right\rangle$ (above $T_{\mathrm{c}}$ ). Here $h^{\text {ext }}$ is an applied conjugate field, $\chi_{0}$ is the response function, and $J\left(R_{j i}\right)$ is an interaction. In other words, the mean field approximation reduces the many-particle problem to a single-site problem in which a magnetic moment at any site can be either parallel or antiparallel to the total magnetic field composed of the applied field and the molecular field. The average interaction of $i$ neighbors was taken into account only, and the fluctuations were neglected. One particular example, where the mean field theory works relatively well is the homogeneous structural phase transitions; in this case the fluctuations 
are confined in phase space. The next important step was made by L. Neel. He conjectured that the Weiss internal field might be either positive or negative in sign. In the latter case, he showed that below a critical temperature (Neel temperature) an ordered arrangement of equal numbers of oppositely directed atomic moments could be energetically favorable. This new magnetic structure was termed antiferromagnetism. It was conjectured that the two-sublattice Neel (classical) ground state is formed by local staggered internal mean fields.

There is a number of "correlated effective field" theories, that tend to repair the limitations of simplified mean field theories. The remarkable and ingenious one is the Onsager "reaction field approximation". He suggested that the part of the molecular field on a given dipole moment which comes from the reaction of neighboring molecules to the instantaneous orientation of the moment should not be included into the effective orienting field. This "reaction field" simply follows the motion of the moment and thus does not favor one orientation over another (for details see [22, 38]).

It is known [56] that mean-field approximations, for example the molecular field approximation for a spin system, the Hartree-Fock approximation and the BCS-Bogolyubov approximation for an electron system are universally formulated by the Bogolyubov inequality:

$$
-\beta^{-1} \ln \left(\operatorname{Tr} \mathrm{e}^{(-\beta H)}\right) \leqslant-\beta^{-1} \ln \left(\operatorname{Tr} \mathrm{e}^{\left(-\beta H^{\mathrm{mf}}\right)}\right)+\frac{\operatorname{Tr} \mathrm{e}^{\left(-\beta H^{\mathrm{mf}}\right)}\left(H-H^{\mathrm{mf}}\right)}{\operatorname{Tr} \mathrm{e}^{\left(-\beta H^{\mathrm{mf}}\right)} .}
$$

Here $F$ is the free energy, and $H^{\mathrm{mf}}$ is a "trial" or a "mean field" approximating Hamiltonian. This inequality gives the upper bound of the free energy of a many-body system. It is important to emphasize that the BCS-Bogolyubov theory of superconductivity [12, 41, 42] was formulated on the basis of a trial Hamiltonian which consists of a quadratic form of creation and annihilation operators, including "anomalous" (off-diagonal) averages. The functional of the mean field (for the superconducting single-band Hubbard model) is of the following form [57]:

$$
\Sigma_{\sigma}^{c}=U\left(\begin{array}{cc}
\left\langle a_{i-\sigma}^{\dagger} a_{i-\sigma}\right\rangle & -\left\langle a_{i \sigma} a_{i-\sigma}\right\rangle \\
-\left\langle a_{i-\sigma}^{\dagger} a_{i \sigma}^{\dagger}\right\rangle & -\left\langle a_{i \sigma}^{\dagger} a_{i \sigma}\right\rangle
\end{array}\right)
$$

The "anomalous" off-diagonal terms fix the relevant BCS-Bogolyubov vacuum and select the appropriate set of solutions. From the point of view of quantum many-body theory, the problem of adequate introduction of mean fields for a system of many interacting particles can be most consistently investigated in the framework of the IGF method. A correct calculation of the quasiparticle spectra and their damping, particularly, for systems with a complicated spectrum and strong interaction [22] reveals, that the generalized mean fields can have very complicated structure which cannot be described by a functional of the mean-particle density.

To illustrate the actual distinction of description of the generalized mean field in the equationof-motion method for the double-time Green functions, let us compare the two approaches, namely, that of Tyablikov [19] and of Callen [58]. We shall consider the Green function $\left\langle\left\langle S^{+} \mid S^{-}\right\rangle\right\rangle$for the isotropic Heisenberg model

$$
H=-\frac{1}{2} \sum_{i j} J(i-j) \vec{S}_{i} \vec{S}_{j}
$$

The equation of motion for the spin Green function is of the form

$$
\omega\left\langle\left\langle S_{i}^{+} \mid S_{j}^{-}\right\rangle\right\rangle_{\omega}=2\left\langle S^{z}\right\rangle \delta_{i j}+\sum_{g} J(i-g)\left\langle\left\langle S_{i}^{+} S_{g}^{z}-S_{g}^{+} S_{i}^{z} \mid S_{j}^{-}\right\rangle\right\rangle_{\omega}
$$

The Tyablikov decoupling expresses the second-order Green function in terms of the first (initial) Green function:

$$
\left\langle\left\langle S_{i}^{+} S_{g}^{z} \mid S_{j}^{-}\right\rangle\right\rangle=\left\langle S^{z}\right\rangle\left\langle\left\langle S_{i}^{+} \mid S_{j}^{-}\right\rangle\right\rangle
$$

This approximation is an RPA-type; it does not lead to the damping of spin wave excitations

$$
E(q)=\sum_{g} J(i-g)\left\langle S^{z}\right\rangle \exp \left[\mathrm{i}\left(\vec{R}_{i}-\vec{R}_{g}\right) \vec{q}\right]=2\left\langle S^{z}\right\rangle\left(J_{0}-J_{q}\right)
$$


The reason for this is rather transparent. This decoupling does not take into account the inelastic magnon-magnon scattering processes. In a sense, the Tyablikov approximation consists of approximating the commutation relations of spin operators to the extent of replacing the commutation relation $\left[S_{i}^{+}, S_{j}^{-}\right]_{-}=2 S_{i}^{z} \delta_{i j}$ by $\left[S_{i}^{+}, S_{j}^{-}\right]_{-}=2\left\langle S^{z}\right\rangle \delta_{i j}$.

Callen [58] has proposed an improved decoupling approximation in the method of Tyablikov in the following form:

$$
\left\langle\left\langle S_{g}^{z} S_{f}^{+} \mid B\right\rangle\right\rangle \rightarrow\left\langle S^{z}\right\rangle\left\langle\left\langle S_{f}^{+} \mid B\right\rangle\right\rangle-\alpha\left\langle S_{g}^{-} S_{f}^{+}\right\rangle\left\langle\left\langle S_{g}^{+} \mid B\right\rangle\right\rangle .
$$

Here $0 \leqslant \alpha \leqslant 1$. To clarify this point, it should be reminded that for spin $1 / 2$ ( the procedure was generalized by Callen to an arbitrary spin), the spin operator $S^{z}$ can be written as $S_{g}^{z}=S-S_{g}^{-} S_{g}^{+}$ or $S_{g}^{z}=\frac{1}{2}\left(S_{g}^{+} S_{g}^{-}-S_{g}^{-} S_{g}^{+}\right)$. It is easy to show that

$$
S_{g}^{z}=\alpha S+\frac{1-\alpha}{2} S_{g}^{+} S_{g}^{-}-\frac{1+\alpha}{2} S_{g}^{-} S_{g}^{+}
$$

The operator $S_{g}^{-} S_{g}^{+}$represents the deviation of $\left\langle S^{z}\right\rangle$ from $S$. In the low-temperature region, this deviation is small, and $\alpha \sim 1$. Similarly, the operator $\frac{1}{2}\left(S_{g}^{+} S_{g}^{-}-S_{g}^{-} S_{g}^{+}\right)$represents the deviation of $\left\langle S^{z}\right\rangle$ from 0 . Thus, when $\left\langle S^{z}\right\rangle$ approaches zero, one can expect that $\alpha \sim 0$. Thus, in this way, it is possible to obtain a correction to the Tyablikov decoupling with either a positive or negative sign, or no correction at all, or any intermediate value, depending on the choice of $\alpha$. The above Callen arguments are not rigorous, for, although the difference in the operators $S^{+} S^{-}$and $S^{-} S^{+}$is small if $\left\langle S^{z}\right\rangle \sim 0$, each operator makes a contribution of the order of $S$, and it is each operator which is treated approximately, not the difference. There are some other drawbacks of the Callen decoupling scheme. Nevertheless, the Callen decoupling was the first conceptual attempt to introduce the interpolation decoupling procedure. Let us note that the choice of $\alpha=0$ over the entire temperature range is just the Tyablikov decoupling (27).

The energy spectrum for the Callen decoupling is given by

$$
E(q)=2\left\langle S^{z}\right\rangle\left(\left(J_{0}-J_{q}\right)+\frac{\left\langle S^{z}\right\rangle}{N S^{2}} \sum_{k}[J(k)-J(k-q)] N(E(k))\right) .
$$

Here $N(E(k))$ is the Bose distribution function $N(E(k))=[\exp (E(k) \beta)-1]^{-1}$. This is an implicit equation for $N(E(k))$, involving the unknown quantity $\left\langle S^{z}\right\rangle$. For the latter an additional equation is given [58]. Thus, both these equations constitute a set of coupled equations which should be solved self-consistently for $\left\langle S^{z}\right\rangle$. This formulation of the Callen decoupling scheme explicitly displays the tendency of the improved description of the mean field. In a sense, it is possible to say that the Callen work dates really the idea of the generalized mean field within the equation-of-motion method for double-time GFs, although, in a semi-intuitive form. The next essential steps were made by Plakida [53] for the Heisenberg ferromagnet and by Kuzemsky [30] for the Hubbard model. Later many approximate schemes for decoupling the hierarchy of equations for GF were proposed, improving the Tyablikov's and Callen's decouplings. Various approaches generalizing the approximation of a random phase in the ferromagnetism theory for wide ranges of temperature were considered in the paper by Czachor and Holas [59]. As was mentioned above, the correct definition of generalized mean fields depends on the condition of the problem, the strength of interaction, the choice of relevant operators, and on the symmetry requirements. The most important conclusion to be drawn from the present consideration is that the GMF, in principle, can have quite a nontrivial structure and cannot be reduced to the mean-density functional only.

\section{Quasiaverages and irreducible Green functions method}

In condensed matter physics, the symmetry is important in classifying different phases and in understanding the phase transitions occurring between them. There is an important distinction between the case where the broken symmetry is continuous (e.g. translation, rotation, gauge invariance) or discrete (e.g., inversion, time reversal symmetry) [22]. The Goldstone theorem states that when a continuous symmetry is spontaneously broken and the interactions are short ranged, 
a collective mode (excitation) exists with a gapless energy spectrum (i.e., the energy dispersion curve starts at zero energy being continuous). Acoustical phonons in a crystal are prime examples of the so-called gapless Goldstone modes. Other examples are the Bogolyubov sound modes in (charge neutral) Bose condensates [43 45] and spin waves (magnons) in ferro- and antiferromagnets. N.N. Bogolyubov and then Y. Nambu in their works showed that the general features of superconductivity are in fact model independent consequences of spontaneous breakdown of electromagnetic gauge invariance. It is important to emphasize that the BCS-Bogolyubov theory of superconductivity [12, 41, 42] was formulated on the basis of a trial Hamiltonian which consists of a quadratic form of creation and annihilation operators, including "anomalous" (off-diagonal) averages [13]. The strong-coupling BCS-Bogolyubov theory of superconductivity was formulated for the Hubbard model in the localized Wannier representation in [57, 60, 61]. Therefore, instead of the algebra of the operator of a normal state $a_{i \sigma}, a_{i \sigma}^{\dagger}$ and $n_{i \sigma}$, for description of superconducting states, one has to use a more general algebra, which includes the operators $a_{i \sigma}, a_{i \sigma}^{\dagger}, n_{i \sigma}$ and $a_{i \sigma} a_{i-\sigma}, a_{i \sigma}^{\dagger} a_{i-\sigma}^{\dagger}$. The relevant generalized one-electron Green function will have the following form [38, 57, 60]:

$$
G_{i j}(\omega)=\left(\begin{array}{ll}
G_{11} & G_{12} \\
G_{21} & G_{22}
\end{array}\right)=\left(\begin{array}{cc}
\left\langle\left\langle a_{i \sigma} \mid a_{j \sigma}^{\dagger}\right\rangle\right\rangle & \left\langle\left\langle a_{i \sigma} \mid a_{j-\sigma}\right\rangle\right\rangle \\
\left\langle\left\langle a_{i-\sigma}^{\dagger} \mid a_{j \sigma}^{\dagger}\right\rangle\right\rangle & \left\langle\left\langle a_{i-\sigma}^{\dagger} \mid a_{j-\sigma}\right\rangle\right\rangle
\end{array}\right) .
$$

As it was discussed in 22, 38], the off-diagonal (anomalous) entries of the above matrix select the vacuum state of the system in the BCS-Bogolyubov form, and they are responsible for the presence of anomalous averages. To treat the problem, we follow the general scheme of irreducible Green functions method [22, 38]. In this approach we start with the equation of motion for the Green function $G_{i j}(\omega)$ (normal and anomalous components)

$$
\begin{aligned}
\sum_{j}\left(\omega \delta_{i j}-t_{i j}\right)\left\langle\left\langle a_{j \sigma} \mid a_{i^{\prime} \sigma}^{\dagger}\right\rangle\right\rangle & =\delta_{i i^{\prime}}+U\left\langle\left\langle a_{i \sigma} n_{i-\sigma} \mid a_{i^{\prime} \sigma}^{\dagger}\right\rangle\right\rangle+\sum_{n j} V_{i j n}\left\langle\left\langle a_{j \sigma} u_{n} \mid a_{i^{\prime} \sigma}^{\dagger}\right\rangle\right\rangle, \\
\sum_{j}\left(\omega \delta_{i j}+t_{i j}\right)\left\langle\left\langle a_{j-\sigma}^{\dagger} \mid a_{i^{\prime} \sigma}^{\dagger}\right\rangle\right\rangle & =-U\left\langle\left\langle a_{i-\sigma}^{\dagger} n_{i \sigma} \mid a_{i^{\prime} \sigma}^{\dagger}\right\rangle\right\rangle+\sum_{n j} V_{j i n}\left\langle\left\langle a_{j-\sigma}^{\dagger} u_{n} \mid a_{i^{\prime} \sigma}^{\dagger}\right\rangle\right\rangle .
\end{aligned}
$$

The irreducible Green functions are introduced by definition

$$
\begin{array}{r}
\left.{ }^{(\mathrm{ir})}\left\langle\left\langle a_{i \sigma} a_{i-\sigma}^{\dagger} a_{i-\sigma} \mid a_{i^{\prime} \sigma}^{\dagger}\right\rangle\right\rangle_{\omega}\right)=\left\langle\left\langle a_{i \sigma} a_{i-\sigma}^{\dagger} a_{i-\sigma} \mid a_{i^{\prime} \sigma}^{\dagger}\right\rangle\right\rangle_{\omega}-\left\langle n_{i-\sigma}\right\rangle G_{11}+\left\langle a_{i \sigma} a_{i-\sigma}\right\rangle\left\langle\left\langle a_{i-\sigma}^{\dagger} \mid a_{i^{\prime} \sigma}^{\dagger}\right\rangle\right\rangle_{\omega}, \\
\left({ }^{\mathrm{ir})}\left\langle\left\langle a_{i \sigma}^{\dagger} a_{i \sigma} a_{i-\sigma}^{\dagger} \mid a_{i^{\prime} \sigma}^{\dagger}\right\rangle\right\rangle_{\omega}\right)=\left\langle\left\langle a_{i \sigma}^{\dagger} a_{i \sigma} a_{i-\sigma}^{\dagger} \mid a_{i^{\prime} \sigma}^{\dagger}\right\rangle\right\rangle_{\omega}-\left\langle n_{i \sigma}\right\rangle G_{21}+\left\langle a_{i \sigma}^{\dagger} a_{i-\sigma}^{\dagger}\right\rangle\left\langle\left\langle a_{i \sigma} \mid a_{i^{\prime} \sigma}^{\dagger}\right\rangle\right\rangle_{\omega} .
\end{array}
$$

The self-consistent system of superconductivity equations follows from the Dyson equation 22 ,, 38 , 57]

$$
\hat{G}_{i i^{\prime}}(\omega)=\hat{G}_{i i^{\prime}}^{0}(\omega)+\sum_{j j^{\prime}} \hat{G}_{i j}^{0}(\omega) \hat{M}_{j j^{\prime}}(\omega) \hat{G}_{j^{\prime} i^{\prime}}(\omega) .
$$

The mass operator $M_{j j^{\prime}}(\omega)$ describes the processes of inelastic electron scattering on lattice vibrations. The elastic processes are described by the quantity (24). Thus the "anomalous" off-diagonal terms fix the relevant BCS-Bogolyubov vacuum and select the appropriate set of solutions. The functional of generalized mean field for superconducting single-band Hubbard model is of the form $\Sigma_{\sigma}^{c}$. A remark about the BCS-Bogolyubov mean-field approach is instructive. Speaking in physical terms, this theory correctly involves a condensation although such a condensation cannot be obtained by an expansion in the effective interaction between electrons. Other mean field theories, e.g. the Weiss molecular field theory and the van der Waals theory of the liquid-gas transition are much less reliable. The reason why a mean-field theory of the superconductivity in the BCS-Bogolyubov form is successful would appear to be that the main correlations in metal are governed by the extreme degeneracy of the electron gas. The correlations due to the pair condensation, although they have dramatic effects, are weak (at least in the ordinary superconductors) in comparison with the typical electron energies, and may be treated in an average way with a reasonable accuracy. It should be emphasized that the high-temperature superconductors discovered two decades ago 
motivated an intensification of research in superconductivity, not only because applications are promising, but because they also represent a new state of matter that breaks certain fundamental symmetries. These are the broken symmetries of gauge (superconductivity), reflection ( $d$-wave superconducting order parameter), and time-reversal (ferromagnetism).

Both superconductivity and antiferromagnetism are the spontaneously broken symmetries. The question of symmetry breaking within the localized and band models of antiferromagnets was studied by the author of this work in 22, 32, 37, 51]. It has been found therein that the concept of spontaneous symmetry breaking in the band model of magnetism is much more complicated than in the localized model. In the framework of the band model of magnetism one has to additionally consider the so-called anomalous propagators of the form

$\mathrm{FM}: \quad G_{\mathrm{fm}} \sim\left\langle\left\langle a_{k \sigma} ; a_{k-\sigma}^{\dagger}\right\rangle\right\rangle$,

$\mathrm{AFM}: \quad G_{\mathrm{afm}} \sim\left\langle\left\langle a_{k+Q \sigma} ; a_{k+Q^{\prime} \sigma^{\prime}}^{\dagger}\right\rangle\right\rangle$.

In the case of the band antiferromagnet, the ground state of the system corresponds to a spindensity wave (SDW), where a particle scattered on the internal inhomogeneous periodic field gains the momentum $Q-Q^{\prime}$ and changes its spin: $\sigma \rightarrow \sigma^{\prime}$. The long-range order parameters are defined as follows:

$$
\begin{gathered}
\mathrm{FM}: m=1 / N \sum_{k \sigma}\left\langle a_{k \sigma}^{\dagger} a_{k-\sigma}\right\rangle, \\
\mathrm{AFM}: M_{Q}=\sum_{k \sigma}\left\langle a_{k \sigma}^{\dagger} a_{k+Q-\sigma}\right\rangle .
\end{gathered}
$$

It is important to stress that the long-range order parameters here are functionals of the internal field, which in turn is a function of the order parameter. Thus, in the cases of rotation and translation of invariant Hamiltonians of band ferro- and antiferromagnetics, one has to add the following infinitesimal sources removing the degeneracy:

$$
\begin{array}{r}
\mathrm{FM}: \nu \mu_{\mathrm{B}} H_{x} \sum_{k \sigma} a_{k \sigma}^{\dagger} a_{k-\sigma}, \\
\mathrm{AFM}: \nu \mu_{\mathrm{B}} H \sum_{k Q} a_{k \sigma}^{\dagger} a_{k+Q-\sigma} .
\end{array}
$$

Here, $\nu \rightarrow 0$ after the infinite-volume limit $V \rightarrow \infty$ which is usual in statistical mechanics. The ground state in the form of a spin-density wave was obtained for the first time by Overhauser. There, the vector $\vec{Q}$ is a measure of inhomogeneity or translation symmetry breaking in the system. The analysis performed by various authors showed that the antiferromagnetic and more complicated states (for instance, ferrimagnetic) can be described in the framework of a generalized mean-field approximation [37]. In doing that we have to take into account both the normal averages $\left\langle a_{i \sigma}^{\dagger} a_{i \sigma}\right\rangle$ and the anomalous averages $\left\langle a_{i \sigma}^{\dagger} a_{i-\sigma}\right\rangle$. It is clear that the anomalous terms break the original rotational symmetry of the Hubbard Hamiltonian. Thus, the generalized mean-field approximation for an antiferromagnet has the following form 37] $n_{i-\sigma} a_{i \sigma} \simeq\left\langle n_{i-\sigma}\right\rangle a_{i \sigma}-\left\langle a_{i-\sigma}^{\dagger} a_{i \sigma}\right\rangle a_{i-\sigma}$. A selfconsistent theory of band antiferromagnetism 37| was developed by the author of this work using the method of irreducible Green functions [22, 38]. The following definition of irreducible Green functions was used:

$$
\begin{aligned}
{ }^{\mathrm{ir}}\left\langle\left\langle a_{k+p \sigma} a_{p+q-\sigma}^{\dagger} a_{q-\sigma} \mid a_{k \sigma}^{\dagger}\right\rangle\right\rangle_{\omega}= & \left\langle\left\langle a_{k+p \sigma} a_{p+q-\sigma}^{\dagger} a_{q-\sigma} \mid a_{k \sigma}^{\dagger}\right\rangle\right\rangle_{\omega} \\
& -\delta_{p, 0}\left\langle n_{q-\sigma}\right\rangle G_{k \sigma}-\left\langle a_{k+p \sigma} a_{p+q-\sigma}^{\dagger}\right\rangle\left\langle\left\langle a_{q-\sigma} \mid a_{k \sigma}^{\dagger}\right\rangle\right\rangle_{\omega} .
\end{aligned}
$$

The algebra of relevant operators should be chosen as follows $\left(\left(a_{i \sigma}, a_{i \sigma}^{\dagger}, n_{i \sigma}, a_{i \sigma}^{\dagger} a_{i-\sigma}\right)\right.$. The corresponding initial GF will have the following matrix structure

$$
\mathcal{G}_{\mathrm{AFM}}=\left(\begin{array}{cc}
\left\langle\left\langle a_{i \sigma} \mid a_{j \sigma}^{\dagger}\right\rangle\right\rangle & \left\langle\left\langle a_{i \sigma} \mid a_{j-\sigma}^{\dagger}\right\rangle\right\rangle \\
\left\langle\left\langle a_{i-\sigma} \mid a_{j \sigma}^{\dagger}\right\rangle\right\rangle & \left\langle\left\langle a_{i-\sigma} \mid a_{j-\sigma}^{\dagger}\right\rangle\right\rangle
\end{array}\right) .
$$


The off-diagonal terms select the vacuum state of the antiferromagnet of the band in the form of a spin-density wave. With this definition, one introduces the so-called anomalous (off-diagonal) Green functions which fix the relevant vacuum and select the proper symmetry broken solutions. The theory of itinerant antiferromagnetism [37] was formulated by using sophisticated arguments of irreducible Green functions method in complete analogy with our description of the Heisenberg antiferromagnet at finite temperatures [32]. For a two-sublattice antiferromagnet we used the matrix Green function of the form

$$
\hat{G}(k ; \omega)=\left(\begin{array}{ll}
\left\langle\left\langle S_{k a}^{+} \mid S_{-k a}^{-}\right\rangle\right\rangle & \left\langle\left\langle S_{k a}^{+} \mid S_{-k b}^{-}\right\rangle\right\rangle \\
\left\langle\left\langle S_{k b}^{+} \mid S_{-k a}^{-}\right\rangle\right\rangle & \left\langle\left\langle S_{k b}^{+} \mid S_{-k b}^{-}\right\rangle\right\rangle
\end{array}\right) .
$$

Here, the Green functions on the main diagonal are the usual or normal Green functions, while the off-diagonal Green functions describe contributions from the so-called anomalous terms, analogous to the anomalous terms in the BCS-Bogolyubov superconductivity theory. The anomalous (or offdiagonal) average values in this case select the vacuum state of the system precisely in the form of the two-sublattice Neel state [22].

\section{Conclusions}

In the present work we showed that the development and improvement of the methods of quantum statistical mechanics still remain quite an important direction of research. In particular, the Bogolyubov's method of quasiaverages provides a profound foundation and clarification of the concept of broken symmetry 62. It makes the emphasis on the notion of a degeneracy and plays an important role in equilibrium statistical mechanics of many-particle systems. According to that concept, infinitely small perturbations can trigger macroscopic responses in the system if they break some symmetry and remove the related degeneracy (or quasidegeneracy) of the equilibrium state. As a result, they can produce macroscopic effects even when the perturbation magnitude tends to zero, provided this happens after passing to the thermodynamic limit.

We have discussed the theory of correlation effects for many-particle interacting systems using the ideas of quasiaverages for interacting electron and spin systems on a lattice. The workable and self-consistent irreducible Green functions approach to the decoupling problem for the equationof-motion method for double-time temperature Green functions has been presented. The main advantage of the formalism consists in the clear separation of the elastic scattering corrections (generalized mean fields) and inelastic scattering effects (damping and finite lifetimes). These effects could be self-consistently incorporated in a general and compact manner. Using the IGF method, it is possible to obtain a closed self-consistent set of equations determining the relevant Green functions and self-energy. These equations give a general microscopic description of correlation effects. Moreover, this approach provides a workable scheme for the definition of relevant generalized mean fields written in terms of appropriate correlators. This picture of interacting many-particle systems on a lattice is far richer and presents more possibilities for the analysis of the phenomena that can actually occur. In this sense, the approach we described produces more advanced physical picture of the quasiparticle many-body dynamics.

\section{References}

1. Anderson P.W., Basic Notions of Condensed Matter Physics. W.A. Benjamin, New York, 1984.

2. Rosen J., Symmetry Rules. Springer, Berlin, 2008.

3. Barone M., Theophilou A.K., J. Phys. Conf. Ser., 2008, 104, 012037.

4. Wilczek F.J., Nature, 2005, 433, 239.

5. Strocchi F., Symmetry Breaking. Springer, Berlin, 2005.

6. Nambu Y., J. Phys. Soc. Jpn., 2007, 76, 111002.

7. Nambu Y., Int. J. Mod. Phys. A, 2009, 24, 2371.

8. Grib A.A., Problem of Noninvariant Vacuum in Quantum Field Theory. Atomizdat, Moscow, 1978 (in Russian).

9. Bogolyubov N.N., Physica, 1960, 26 Suppl., S1. 
10. Bogolyubov N.N., Quasiaverages in Problems of Statistical Mechanics. Communication JINR D-781, JINR, Dubna, 1961 (in Russian).

11. Bogolyubov N.N., On the Principle of the Weakening of Correlations in the Method of Quasiaverages. Communication JINR P-549, JINR, Dubna, 1961 (in Russian).

12. Bogolyubov N.N., Sov. J. Part. Nucl., 1971, 1, 1.

13. Bogolyubov N.N., Bogolyubov N.N. (Jr.), Introduction to Quantum Statistical Mechanics, 2nd ed. World Scientific, Singapore, 2009.

14. Bogolyubov N.N. (Jr.), Method for Studying Model Hamiltonians. Pergamon, Oxford, 1972.

15. Bogolyubov N.N. (Jr.), J. Math. Phys., 1973, 14, 79.

16. Bogolyubov N.N. (Jr.), Sadovnikov B.I., Some Problems in Statistical Mechanics. Vyssh. Shkola, Moscow, 1975 (in Russian).

17. Bogolyubov N.N. (Jr.), Sadovnikov B.I., Schumovsky A.S., Mathematical Methods for Statistical Mechanics of Model Systems. Nauka, Moscow, 1989 (in Russian).

18. Petrina D.Ya., Mathematical Foundations of Quantum Statistical Mechanics. Kluwer Academic Publ., Dordrecht, 1995.

19. Tyablikov S.V., Methods in the Quantum Theory of Magnetism. Plenum Press, New York, 1967.

20. Zubarev D.N., Nonequilibrium Statistical Thermodynamics. Consultant Bureau, New-York, 1974.

21. Kuzemsky A.L., Int. J. Mod. Phys. B, 2007, 21, 2831.

22. Kuzemsky A.L., Phys. Part. Nuclei, 2009, 40, 949.

23. Kuzemsky A.L., Int. J. Mod. Phys. B, 2010, 24, 835.

24. Sadovnikov B.I., Fedyanin V.K., Theor. Math. Phys., 1973, 16, 368 (in Russian).

25. Laughlin R.B., Pines D., Proc. Natl. Acad. Sci. U.S.A., 2000, 97, 28.

26. Cox D.L., Pines D., MRS Bulletin, 2005, 30, 425.

27. Levin M., Wen X.-G., Rev. Mod. Phys., 2005, 77, 871.

28. Kuzemsky A.L., Works on Statistical Physics and Quantum Theory of Solid State. JINR Publishing, Dubna, 2009 (in Russian).

29. Bogolyubov N.N., Tyablikov S.V., Doklady Acad. Nauk SSSR, 1959, 126, 53 (in Russian).

30. Kuzemsky A.L., Theor. Math. Phys., 1978, 36, 208 (in Russian).

31. Kuzemsky A.L., Sov. Phys. Dokl., 1989, 34, 974.

32. Kuzemsky A.L., Marvakov D., Theor. Math. Phys., 1990, 83, 147 (in Russian).

33. Kuzemsky A.L., Nuovo Ciment. B, 1994, 109, 829.

34. Czycholl G., Kuzemsky A.L., Wermbter S., Europhysics Lett., 1996, 34, 133.

35. Kuzemsky A.L., Int. J. Mod. Phys. B, 1996, 10, 1895.

36. Kuzemsky A.L., Int. J. Mod. Phys., B, 1999, 13, 2573.

37. Kuzemsky A.L., Physica A, 1999, 267, 131.

38. Kuzemsky A.L., Rivista del Nuovo Cimento, 2002, 25, 1.

39. Kuzemsky A.L., Int. J. Mod. Phys., B, 2004, 18, 3227.

40. Kuzemsky A.L., Physica B, 2005, 355, 318.

41. Bogolyubov N.N., Nuovo Cimento, 1958, 7, 794.

42. Bogolyubov N.N., Tolmachev V.V., Shirkov D.V., A New Method in the Theory of Superconductivity. Consultant Bureau, New-York, 1958.

43. Pitaevskii L., Stringari S., Bose-Einstein Condensation. Oxford University Press, Oxford, 2003.

44. Pethick C.J., . Smith H., Bose-Einstein Condensation in Dilute Gases. Cambridge University Press, Cambridge, 2002.

45. Griffin A., Nikuni T., Zaremba E., Bose-Condensed Gases at Finite Temperatures. Cambridge University Press, Cambridge, 2009.

46. Kovalevsky M. Yu., Peletminskii S.V., Statistical Mechanics of Quantum Liquids and Crystals. Fizmatlit, Moscow, 2006 (in Russian).

47. Peletminskii S.V., Sokolovskii A.I., Theor. Math. Phys., 1974, 18, 121 (in Russian).

48. Vozyakov V.I., Theor. Math. Phys., 1979, 39, 129 (in Russian).

49. Peregoudov D.V., Theor. Math. Phys., 1997, 113, 149 (in Russian).

50. Bogolyubov N.N. (Jr.), Demyanenko D.A., Kovalevsky M.Yu., Chekanova N.N., Phys, Atom. Nucl., 2009, 72, 761 .

51. Kuzemsky A.L., Fundamental Principles of the Physics of Magnetism and the Problem of Itinerant and Localized Electronic States. Communication JINR E17-2000-32, Dubna, 2000.

52. Kuzemsky A.L., Int. J. Mod. Phys. B, 2002, 16, 803.

53. Plakida N.M., Phys. Lett. A, 1973, 43, 481.

54. Lee M.H., Phys. Rev. E, 2000, 61, 3571. 
55. Balucani U., Lee M.H., Tognetti V. Phys. Rep., 2003, 373, 409.

56. Soldatov A.V., Phys. Part. Nuclei B, 2000, 31, No. 7, 138.

57. Vujicic G., Kuzemsky A.L., Plakida N.M., Theor. Math. Phys., 1982, 53, 138 (in Russian).

58. Callen H.B., Phys. Rev., 1963, 130, 890.

59. Czachor A., Holas A., Phys. Rev. B, 1990, 41, 4674.

60. Kuzemsky A.L., Holas A., Plakida N.M., Physica B, 1983, 122, 168.

61. Wysokinski K., Kuzemsky A.L., J. Low Temp. Phys., 1983, 52, 81.

62. Bogolyubov, N.N. (Jr.), Sankovich D.P., Usp. Mat. Nauk., 1994, 49, 21 (in Russian).

\title{
Квазісередні, порушення симетрії і метод незвідних функцій Гріна
}

\author{
А.Л. Куземський \\ Лабораторія теоретичної фізики ім. Боголюбова, Об'єднаний інститут ядерних досліджень, \\ Дубна, Росія
}

\begin{abstract}
Розглянуто розвиток і застосування методу квазісередніх у квантовій статистичній фізиці і квантовій теорії твердого тіла, зокрема, у квантовій теорії магнетизму. Показано, що роль симетрії (i порушення симетрії) у поєднанні з виродженням системи була переглянута і суттєво прояснена завдяки методу квазісередніх. Проблема знаходження феромагнітного, антиферомагнітного і надпровідного розв'язків “порушення симетрії” скорельованих ґраткових ферміонних моделей обговорена в рамках методу функцій Гріна. Уніфікована схема побудови узагальнених середніх полів (поправки пружного розсіяння) і власної енергії (непружного розсіяння) в термінах рівнянь руху і рівняння Дайсона узагальнена з метою включення “полів джерел”. Цей підхід доповнює попередні дослідження мікроскопічної теорії антиферомагнетизму і прояснює концепції підґраток Нееля для локалізованого і колективного антиферомагнетизму і “спін-вирівнюючих полів" скорельованих ґраткових ферміонів.
\end{abstract}

Ключові слова: квантова статистична механіка, квазісередні Боголюбова, порушення симетрії, квантова теорія магнетизму, мікроскопічна теорія надпровідності 